Revista Signos

2008, 41(68)

439-457

\title{
Un análisis de la discusión acerca de temas controversiales en Enseñanza Media desde la pragma-dialéctica*
}

\author{
Ana María Vicuña \\ Pontificia Universidad \\ Católica de Chile \\ Chile
}

\author{
Juana Marinkovich \\ Pontificia Universidad \\ Católica de Valparaíso \\ Chile
}

Resumen: El propósito de este estudio es analizar las dificultades que surgen en la argumentación oral en el aula al abordar problemas éticos controversiales, como es el caso de la legalización de la eutanasia en Chile. El corpus de trabajo está constituido por los turnos de habla de los estudiantes que participan en un debate simulado sobre la eutanasia en una clase de Lengua Castellana y Comunicación de tercer año medio de un colegio particular-subvencionado de la ciudad de Valparaíso (Chile). Todo esto en el marco de la unidad de aprendizaje 'La argumentación'. Para mostrar los problemas que los estudiantes enfrentan a la hora de argumentar oralmente, analizamos la estructura argumentativa de sus intervenciones, utilizando el enfoque pragma-dialéctico, y comparamos los argumentos esgrimidos por los estudiantes con las argumentaciones más frecuentes que circulan en el ámbito de la filosofía especializada acerca de la temática en cuestión. Los resultados de dicho análisis evidencian los escollos que los estudiantes enfrentan para conducir una 'discusión crítica', pero al mismo tiempo dan cuenta de un vacío en la formación de los profesores, quienes no están preparados para distinguir entre una argumentación ética o deontológica y otra de tipo pragmática.

Palabras Clave: Pragma-dialéctica, discusión crítica, argumentación ética, argumentación pragmática, eutanasia.

Recibido: 28-XII-2007 Aceptado: 25-VII-2008

\section{* Proyecto FONDECYT 1060439}

Correspondencia: Ana María Vicuña (amvicuna@uc.cl). Departamento de Ciencias del Lenguaje, Facultad de Letras, Pontificia Universidad Católica de Chile. Casilla 114-D. Vicuña Mackenna 4860, Macul, Santiago, Chile. 


\title{
A pragma-dialectical analysis of the debate about controversial issues in high school contexts
}

\begin{abstract}
This article deals with the difficulties that may arise when classroom oral argumentation focuses on controversial ethical issues, such the legalization of euthanasia in Chile. In analyzing the turn-takings of an $11^{\text {th }}$-grade Spanish Language and Communication class in a simulated debate about euthanasia, while the learning unit "Argumentation" was being covered, it was observed that the students found hard to grasp the most complex concepts and to establish the required distinctions. To identify the problems that the students encounter when they had to argue orally, the argumentative structure of their turn-takings was analyzed, using the pragma-dialectical approach, and the students' arguments were compared with the most frequent philosophical arguments on this issue. The findings show the students' pitfalls in conducting a "critical discussion" and, at the same time, they account for the teachers' inexperience to distinguish between an ethical (or deontological) argumentation and a pragmatic argumentation.
\end{abstract}

Key Words: Pragma-dialectics, critical discussion, ethical argumentation, pragmatic argumentation, euthanasia.

\section{INTRODUCCIÓN}

La enseñanza de la argumentación en el sistema escolar chileno se materializa en tercer año medio en la asignatura de Lengua Castellana y Comunicación, principalmente en la unidad de aprendizaje 'La argumentación'. Si revisamos los objetivos fundamentales de dicho nivel, observamos que de seis objetivos, cuatro de ellos apuntan al discurso argumentativo, tal como lo expresa el programa de tercer año medio de la asignatura en cuestión:

"(1) Comprender los procesos de comunicación centrados en la controversia generada por diferencias de opinión y el discurso argumentativo propio de esas situaciones; (2) Reconocer y utilizar con propiedad los principales elementos, recursos y procedimientos del discurso argumentativo oral y escrito; (3) Fortalecer el respeto por los puntos de vista divergentes, valorando sus aportes y alcanzando una evaluación crítica de la validez de los argumentos propios y ajenos; apreciar el aporte de estas actitudes para la formación personal y la convivencia democrática; (4) Afianzar el dominio de las estructuras gramaticales y textuales pertinentes al discurso argumentativo, así como del léxico y la ortografía" (MINEDUC, 1998: 89).

Estos objetivos están en íntima relación con los contenidos mínimos obligatorios que en el caso de la Comunicación oral se orientan al:

"(1) Análisis de situaciones de interacción comunicativa oral de tipo argumentativo (debates, polémicas, discusiones grabadas de radio o televisión) para percibir: a. temas polémicos, socialmente relevantes y las diferentes posiciones que se manifiestan acerca de ellos; b. la estructura de los discursos (supuestos, argumentos, conclusiones), sus 
procedimientos (tipos de argumentos, validez de ellos, etc.) y los resultados y efectos. (2) Participación en situaciones de interacción comunicativa oral de tipo argumentativo, dando oportunidad para: a. la elección de un punto de vista personal respecto de algún tema polémico; la estructuración de la argumentación correspondiente y el desempeño adecuado en el debate o discusión (respeto de turnos, uso adecuado de niveles de habla y de recursos paraverbales y no verbales, etc.); b. la evaluación de resultados (por ejemplo, derogación de los argumentos de la(s) otra(s) posición(es), reconocimiento del propio error, construcción de consensos, etc.)" (MINEDUC, 1998: 92).

En este contexto, se hace necesario conocer y describir cómo los estudiantes hacen suyos estos objetivos y contenidos en el ámbito de la comunicación oral, tarea que un grupo de investigadores ha intentado llevar a cabo mediante el proyecto denominado "La competencia argumentativa oral en el aula: Un estudio exploratorio con estudiantes de enseñanza media" (FONDECYT 1060439). A modo de avance de algunos resultados obtenidos en este proyecto, luego de la observación y videograbación de 16 sesiones en un establecimiento educacional particular-subvencionado de la ciudad de Valparaíso, se presenta a continuación el análisis de una clase de 45 minutos desarrollada en un tercer año medio de Lengua Castellana y Comunicación del mismo establecimiento educacional cuando los estudiantes discuten el tema de la legalización de la eutanasia en Chile.

Para ello, en un primer momento se despliega el marco de referencia en el que se reflexiona acerca de lo que los programas de estudio de nuestro sistema escolar plantean en cuanto a la argumentación oral en el aula. También se explica cómo la teoría de la argumentación pragma-dialéctica se constituye en una herramienta analítica para dar cuenta de la calidad de las intervenciones de los estudiantes cuando discuten un tema controversial, como lo es el de la legalización de la eutanasia en Chile. En un segundo momento, se procede al análisis de los turnos de habla de cada participante de la clase, utilizando para ello los conceptos clave del modelo pragma-dialéctico de una 'discusión crítica' (van Eemeren \& Grootendorst, 1992), tales como los puntos de vista, las razones o argumentos que los sustentan, basados tanto en principios éticos como en problemas de índole pragmática, y las etapas de dicha discusión. Finalmente, se presenta la interpretación y comentarios acerca de los resultados obtenidos.

\section{Marco de referencia}

De los objetivos fundamentales y contenidos mínimos obligatorios de tercer año medio de la asignatura de Lengua Castellana y Comunicación, el estudio que nos ocupa tiene directa relación con el de la producción oral de un discurso argumentativo. En este sentido, los programas ponen el acento en el manejo con propiedad de los principales elementos, recursos y procedimientos del discurso argumentativo oral. Este manejo debiera permitir la participación de los estudian- 
tes en situaciones de interacción en donde se elige un punto de vista personal respecto de algún tema polémico, se estructura la argumentación correspondiente y se interviene en un debate o discusión (con respeto de turnos, uso adecuado de niveles de habla y de recursos paraverbales y no verbales, etc.).

Entre las ya clásicas teorías contemporáneas acerca de la argumentación -la nueva retórica de Perelman y Olbrechts-Tyteca (1958), la teoría de la lógica cotidiana de Toulmin (1958) y la argumentación en la lengua de Anscombre y Ducrot (1976), entre otras- destaca la perspectiva pragma-dialéctica de van Eemeren y Grootendorst (1992). Esta última propone un modelo ideal de discusión crítica que permite tanto evaluar como interpretar y orientar la producción del discurso argumentativo. Su aplicación nos permitirá, en consecuencia, determinar en qué medida los estudiantes son capaces de cumplir con los objetivos y contenidos que los programas de estudio propician para una efectiva producción oral del discurso argumentativo en tercer año de enseñanza media en la asignatura de Lengua Castellana y Comunicación.

Más aún, teniendo en consideración que la discusión gira, en este caso, en torno a una controversia sobre la legalización de la eutanasia, la teoría pragma-dialéctica distingue entre diversos tipos de argumento que suelen presentarse en una discusión legal. Por un lado, presenta aquellos argumentos que están basados en principios éticos que, a su vez, se reflejan en las leyes de un país y, por otro lado, aquellos que están orientados a la resolución de problemas pragmáticos y que se basan, por tanto, en el contexto social y político en el cual se intentan llevar a cabo las propuestas y resoluciones legales (Feteris, 2007).

\subsection{La argumentación desde la perspectiva de la pragma-dialéctica}

Desde el año 1984, van Eemeren y el equipo de Amsterdam se interesan en la resolución de las diferencias de opinión mediante el discurso argumentativo. Para la teoría pragma-dialéctica, el discurso, o más bien el texto argumentativo, es la completa constelación de enunciados (orales o escritos) que han sido presentados en defensa de un punto de vista. En esta propuesta, la argumentación es de naturaleza interactiva, un tipo de diálogo o, más precisamente, una 'discusión crítica', es decir, un modelo ideal que permite analizar y evaluar los argumentos en cuanto se orientan a la resolución de una diferencia de opinión y potencia el descubrimiento de las falacias en tanto pasos o movimientos incorrectos que impiden o dificultan la resolución.

Los conceptos clave de esta teoría desde el punto de vista del desarrollo de la discusión crítica son: los 'temas controversiales', es decir, los temas que van a generar la disputa; los 'puntos de vista', enunciados que expresan una concepción que supone una cierta toma de posición en una disputa; las 'razones' o los 'argumentos' mediante los cuales se hace un esfuerzo para defender una posición. Estos conceptos se integran a lo que los propiciadores de esta teoría denominan 
las etapas de una discusión crítica o de la resolución de una controversia, a saber, la 'confrontación', que define la diferencia de opinión; la 'apertura', en donde se establecen los distintos tipos de compromisos que son el punto de partida de una discusión y se asignan los roles de los discutidores; la 'argumentación' o el intercambio de argumentos y reacciones críticas; y, finalmente, el 'cierre' o 'clausura', estadio en que se establece el resultado de la discusión.

Por otra parte, las disputas en el marco de la pragma-dialéctica pueden caracterizarse como disputas 'simples' o 'complejas'. En una disputa simple solo se pone en duda un punto de vista que ha sido presentado, con respecto a una proposición. Las disputas complejas pueden variar de acuerdo a si son 'únicas' o 'múltiples' y 'mixtas' o 'no mixtas'. En una disputa única, el punto de vista cuestionado se relaciona con una sola proposición, en una disputa múltiple, en cambio, el punto de vista cuestionado se relaciona con dos o más proposiciones. Si solo se pone en cuestión un punto de vista positivo, o solo uno negativo, la disputa es no mixta; si, en cambio, se cuestiona tanto un punto de vista positivo como uno negativo, con respecto a la misma proposición, se trata de una disputa mixta. De la combinación de estas categorías surgen los cuatro tipos estándar de disputa: disputa única no mixta, disputa única mixta, disputa múltiple no mixta y disputa múltiple mixta (van Eemeren \& Grootendorst, 2006).

\subsection{La argumentación y las controversias éticas}

Los temas controversiales que se les plantean a los estudiantes para su discusión en el aula, como el de la legalización de la eutanasia en Chile, requieren el esclarecimiento de los aspectos éticos y pragmáticos. Estos últimos se relacionan, en este caso, con el ámbito legal, así como también con el de la enseñanza de la argumentación.

\subsubsection{El caso de la legalización de la eutanasia}

Desde una perspectiva ética, el debate acerca de la eutanasia es complejo. Ya en la antigüedad clásica, el infanticidio, el suicidio y la eutanasia eran comúnmente aceptados. Sin embargo, por influencia del judaísmo y del cristianismo, la vida humana se visualiza como sacrosanta y se considera que causar la muerte de un ser humano es una usurpación de los derechos de Dios de dar o quitar la vida. Los filósofos de la modernidad, tales como Hume, Bentham y Stuart Mill cuestionaron esta postura y, en el siglo XVI, un escritor cristiano como Thomas More, en Utopía, había sugerido el uso de la eutanasia como una solución para los enfermos incurables (Kuhse, 1995).

Por otra parte, el psiquiatra y teólogo Scott Peck (1999:15) plantea una serie de interrogantes en orden a ilustrar la complejidad del problema de la eutanasia: 
"¿La eutanasia es solamente un acto que alguien comete -un médico o un miembro de la familia - con una persona enferma o moribunda? ¿O puede también utilizarse la palabra para definir a alguien que está enfermo o moribundo se suicida sin la ayuda de otra persona? ¿La eutanasia requiere el consentimiento del paciente? ¿De la familia? ¿Es diferente a otras formas de suicidio y homicidio? ¿En qué se diferencia del simple hecho de tirar del enchufe? Si un tipo de eutanasia consiste en evitar el uso de recursos heroicos para prolongar la vida, ¿cómo se distinguen los recursos heroicos y aquellos que forman parte del tratamiento? ¿Puede establecerse una diferencia entre el dolor físico y el dolor emocional? Y por encima de todo, ¿por qué hay involucradas cuestiones éticas, y cuáles pueden ser?".

En orden a resolver la controversia ética que subyace tras la eutanasia, Kuhse (1995) introduce algunas distinciones. Una de ellas es la diferencia entre eutanasia voluntaria, no voluntaria e involuntaria, entendiendo a la primera cuando es realizada por $A$ ante la solicitud de $B$ y para el propio bien de $B$, en tanto que la no voluntaria ocurre cuando una persona no ha podido expresar su deseo de morir. En el caso de la eutanasia involuntaria, esta se presenta cuando el consentimiento no se ha dado, o porque no ha sido solicitado o porque ha sido denegado cuando se solicitó. Otra distinción es aquella que se hace entre eutanasia activa y pasiva, dado que los tres tipos mencionados más arriba pueden ser activos o pasivos, porque $A$ puede causar la muerte de $B$ de dos maneras: matando a $B$ o permitiendo que $B$ muera. Si A mata a $B$ administrándole, por ejemplo, una inyección letal, A realiza una eutanasia activa; si A permite que $B$ muera, suspendiendo o negando el tratamiento que mantiene vivo a $B, A$ realiza una eutanasia pasiva.

Por su parte, la Iglesia Católica define la eutanasia como una acción u omisión que ocasiona por sí misma o intencionadamente la muerte (Seper, 1980).

Por último, en Chile, el debate se ha centrado, más bien, en lo legal. Prueba de ello, es el proyecto de ley acerca de la legalización de la eutanasia que se encuentra en la Comisión de Salud del Congreso desde el 2006 y que se funda expresamente en los siguientes principios:

- Derecho a la muerte digna: libertad para determinar terapias, derecho a no padecer males y dolores innecesarios.

- Ciencia y tecnología no deben utilizarse para prolongar artificialmente la vida.

- Toda persona puede manifestar su voluntad o nombrar un curador por cualquier medio que exprese inequívocamente su voluntad.

- Si lo anterior es imposible, los médicos consultarán a los parientes más próximos.

Un examen de estos principios muestra, por una parte, que están basados en consideraciones de tipo ético, relacionadas con la libertad individual y, por otra, en consideraciones prácticas 
relativas a los problemas derivados de la utilización indiscriminada de los avances de la ciencia y tecnología.

\subsubsection{La concepción pragma-dialéctica de la argumentación en la esfera ética}

En un análisis pragma-dialéctico de la argumentación legal, Feteris (2007) enfatiza la necesidad de distinguir claramente entre las argumentaciones de tipo ético y las argumentaciones de naturaleza pragmática. De este modo, extrapolando este análisis a la problemática de la legalización de la eutanasia en Chile, esta debería sustentarse en las siguientes formas básicas (puntos de vista) y plantearse las interrogantes críticas que son pertinentes para cada tipo de argumentación.

- La argumentación pragmática

Punto de vista: Las políticas (gubernamentales) $\mathrm{X}$ son deseables

Porque:

Las políticas (gubernamentales) $\mathrm{X}$ conducen al resultado deseable $\mathrm{Y}$ para que no ocurran los problemas actuales (de políticas, regulación, etc.)

y:

El resultado $Y$ es deseable

\section{Interrogantes críticas}

1. ¿Es correcta la predicción condicional?

(a) ¿Conducen verdaderamente las nuevas políticas $X$ al resultado $Y$ ?

(b) ¿Es el resultado $Y$ en realidad deseable?

(c) ¿Existen otros factores que deban estar presentes en combinación con $\mathrm{X}$ para alcanzar el resultado Y?

(d) ¿Puede el resultado $Y$ obtenerse también con otros medios diferentes a las políticas $X$ ?

- La argumentación ética o deontológica

Punto de vista: Las políticas (gubernamentales) $\mathrm{X}$ son deseables

Porque:

Las políticas (gubernamentales) $\mathrm{X}$ son consistentes con las normas morales $\mathrm{N}$ $\mathrm{y}:$

Las normas morales $\mathrm{N}$ son normas aceptadas 


\section{Interrogantes críticas}

2. ¿Son correctas las políticas $\mathrm{X}$ desde la perspectiva de las normas morales pertinentes N?

(e) ¿Son las políticas $\mathrm{X}$ verdaderamente consistentes con las normas morales N?

(f) ¿Son las normas morales $\mathrm{N}$ aceptadas (dentro de una sociedad en particular)?

Estas distinciones y preguntas deberían aparecer en un debate público sobre la legalización de la eutanasia en Chile, sin embargo, como quedará de manifiesto en el análisis, este tipo de distinciones y preguntas críticas no está en el repertorio de los estudiantes, al menos en el debate que nos ocupa.

\subsubsection{La argumentación ética en contexto escolar}

Si queremos fomentar la 'discusión crítica' en el aula acerca de temas controversiales, como lo es la eutanasia, el programa de Filosofía para Niños (Lipman, Sharp \& Oscanyan, 1980) de amplio reconocimiento en el mundo, plantea el concepto de 'comunidad de indagación', fundada en el diálogo filosófico abierto, autoexigente y crítico de los participantes (estudiantes y profesor/a) sobre temas de su real interés (Vicuña, 1994). En tal contexto, los participantes se involucran activamente en la discusión, pero manteniendo siempre un ambiente de respeto y tolerancia hacia las opiniones divergentes y buscando comprender mejor el problema en cuestión, mediante el aporte cooperativo de todos los involucrados. El objetivo no es ganar una discusión, sino adquirir una mejor comprensión para beneficio de todos. En este proceso se desarrollan innumerables habilidades de razonamiento $\mathrm{y}$, a la vez, conductas y actitudes favorables a la convivencia democrática y al desarrollo de la reflexión ética (Vicuña, López \& Tugendhat, 1997). La argumentación se ejercita y se aprende en este proceso cooperativo y co-constructivo de significado, que implica el ejercicio constante de las habilidades críticas y auto-correctivas de la 'comunidad de indagación', para lo cual se requiere que los participantes estén vitalmente interesados en el tema debatido y que reflexionen sobre sus propias experiencias al respecto. Lamentablemente, esto no ocurre en la sesión de debate que analizaremos a continuación.

\section{El estudio}

\subsection{Objetivo}

En este estudio, se pretende describir los puntos de vista, los argumentos a favor o en contra, como también los principios éticos y los problemas pragmáticos, que despliegan un grupo de estudiantes de tercer año medio de un establecimiento educacional de la ciudad de Valparaíso 
(Chile), cuando participan en un debate ficticio acerca de la legalización de la eutanasia en Chile. Todo esto desde la perspectiva analítica de la teoría pragma-dialéctica de la argumentación. Aún más, el contexto en que este debate se desarrolla, corresponde a una clase de Lengua Castellana y Comunicación, específicamente, en el marco de la Unidad de Aprendizaje 'La argumentación'.

\subsection{Corpus}

El corpus está constituido por la transcripción de 46 turnos de habla generados a partir de un debate ficticio (cfr. anexo), desarrollado durante los 45 minutos que dura la clase, en torno a la legalización de la eutanasia en Chile. En este debate participan solo los alumnos que asumen los roles, previamente asignados, de un sacerdote católico, un psicólogo, un médico, un miembro del Parlamento, un padre de familia, el presidente de una asociación contra la eutanasia y un mediador del debate. Este corpus de trabajo se deriva de un corpus mayor correspondiente a una serie de clases videograbadas y transcritas de Lengua Castellana y Comunicación de dos establecimientos educacionales particular-subvencionados de las ciudades de Valparaíso y Viña del Mar.

\subsection{Metodología de análisis}

De acuerdo con van Eemeren y Grootendorst (2004: 95):

"The aim of the pragma-dialectical analysis is to reconstruct the process of resolving a difference of opinion occurring in an argumentative discourse or text. This means that argumentative reality is systematically analyzed from the perspective of a critical discussion. All components of the discourse or text that are in any way relevant to the resolution are in the reconstruction taken into account; all components that are irrelevant to this concern are left out. In this manner, an analytic reconstruction is given of the argumentative 'deep structure' of the discourse or text".

Siguiendo estas consideraciones, en el análisis de los turnos de habla o intervenciones de los participantes en el debate, determinamos, en primer lugar, los puntos de vista que están en juego, las razones ofrecidas, los roles asumidos, el tipo de discusión o disputa de que se trata y las fases de la resolución del conflicto. Enseguida, se definen los aspectos éticos y pragmáticolegales que sustentan los argumentos desplegados durante el mismo debate y se contrastan con lo que en la argumentación en el ámbito de la filosofía se entiende por eutanasia, como también con las políticas legislativas del país acerca del mismo tema. 


\section{Análisis y discusión de los resultados}

En este apartado se analizan y discuten los resultados en términos de los turnos de habla mediante los cuales los estudiantes expresan sus posiciones acerca de la legalización de la eutanasia en Chile. Todo esto, como se ha dicho, desde la perspectiva analítica de la pragma-dialéctica, es decir, qué puntos de vista se expresan, qué roles adoptan los interlocutores, cómo se representa esquemáticamente la discusión, qué principios éticos y pragmáticos ponen en juego los participantes, qué tipo de disputa y cómo se realizan las etapas de la 'discusión crítica'.

De este modo, encontramos dos puntos de vista sobre la misma proposición, uno positivo y uno negativo:

(1) La eutanasia debería ser legalizada en Chile; y

(2) La eutanasia no debería ser legalizada en Chile

Los estudiantes que asumen los roles del sacerdote católico, de un padre de familia y del presidente de una asociación contra la eutanasia son los protagonistas del punto de vista negativo y los antagonistas del punto de vista positivo, mientras que los estudiantes que representan al psicólogo, al médico y a un miembro del Parlamento, son los protagonistas del punto de vista positivo y los antagonistas del punto de vista negativo. El estudiante que hace de mediador permanece neutral o, en términos de la pragma-dialéctica, adopta un punto de vista cero (van Eemeren \& Grootendorst, 1992).

Dado que los dos puntos de vista que se defienden o se cuestionan en el debate tienen el mismo contenido proposicional (solo que uno es positivo y el otro negativo), la disputa se caracteriza como 'simple y mixta' (van Eemeren \& Grootendorst, 1992). La argumentación para el punto de vista positivo 'La eutanasia debería ser legalizada en Chile', tiene dos argumentos que lo sustentan, con una sub-argumentación en cada caso, y tres contra-argumentos que atacan los argumentos de la parte opuesta. La argumentación para el punto de vista negativo 'La eutanasia no debería ser legalizada en Chile', tiene cuatro argumentos de sustento, dos de los cuales se apoyan en una sub-argumentación.

En la siguiente representación esquemática del análisis del debate (ver Figura 1), PV significa punto de vista; $\mathrm{PV}+$, punto de vista positivo; PV-, punto de vista negativo; R, razón o argumento; entre corchetes, la razón o argumento implícito; y los números árabes indican el número secuencial de la razón o argumento. Se utilizan las letras minúsculas para mostrar las alternativas en relación con una razón o argumento. La sub-argumentación se indica colocando un punto, seguido por números, de acuerdo con el número de los argumentos de sustento. 


\section{PV+ La eutanasia debería ser legalizada en Chile}

R. 1 La eutanasia mejora la calidad de vida.

R.1.1 La eutanasia evita el sufrimiento de la enfermedad tanto al enfermo como a sus familiares.

R.2 La eutanasia evita los excesivos gastos en salud.

R.2.1 El dinero que se está gastando en quienes no pueden ser salvados podría ser usado para salvar a aquellos que sí pueden ser sanados o para incrementar el 'plan AUGE'.

R.3 Desconectar a alguien de una máquina no es lo mismo que matar (contra-argumento).

R.3.1 Hace cien años no existía la tecnología que está disponible hoy y los pacientes terminales morían de forma natural.

R.4 No legalizar la eutanasia prolonga la vida agónica de las personas que están sufriendo y también el sufrimiento de sus familiares (contra-argumento).

R.5 Prolongar la vida más de lo que nuestros cuerpos pueden vivir no es divino sino humano.

R.5.1 Dios nos ha dado la vida hasta que nuestros cuerpos puedan resistir.

\section{PV- La eutanasia no debería ser legalizada en Chile}

R.1 La eutanasia es una falsa compasión.

R.1.1 La eutanasia no permite valorar lo purificatorio y meritorio de la enfermedad.

R.2 [La eutanasia no permite deshacer lo hecho aunque uno se arrepienta]

R.2.1a Es muy posible que quien autorice la eutanasia lamente luego su decisión.

R.2.1b La eutanasia es irreversible.

R.3 La eutanasia está contra las leyes de Dios.

R.3.1a La eutanasia es matar a un ser humano.

R.3.1b Matar es contra la ley de Dios.

Figura 1. Representación pragma-dialéctica del análisis del debate acerca de la legalización de la eutanasia en Chile.

Ahora bien, a partir del análisis pragma-dialéctico precedente, podemos inferir los siguientes principios éticos que están en juego en las argumentaciones de los estudiantes:

- Se debe mejorar la calidad de vida de las personas.

- Se debe evitar el sufrimiento.

- Jamás se debe quitar la vida a una persona.

- La vida es un don divino que solamente a Dios compete dar y quitar.

- No siempre debe evitarse el sufrimiento; es posible darle un valor purificatorio o meritorio.

Paralelamente, se observan en las mismas argumentaciones los siguientes problemas pragmáticos: 
- No parece eficiente, en términos de costo-beneficio, mantener con vida a una persona desahuciada.

- El gasto en personas sin esperanza de salvación es innecesario.

- El dinero podría invertirse mejor en resolver otros problemas de salud de la población.

- La irreversibilidad de la muerte plantea un problema a quienes se arrepienten de haber autorizado la eutanasia.

A su vez, estos principios éticos y problemas pragmáticos surgidos durante el debate, se insertan en las diversas etapas de la 'discusión crítica' (confrontación, apertura y argumentación). En cuanto a la confrontación, más bien pseudo-confrontación, el estudiante que asume el rol de mediador ofrece una definición de 'eutanasia', esto es, "el acto de terminar con la vida de una persona, con su consentimiento o no, con el propósito de minimizar el dolor que esta persona sufre, que se encuentra en un estadio terminal de la enfermedad". Esta definición podría considerarse como un punto de partida, aunque ningún panelista se refiere a ella, y, además, no toma en cuenta las distinciones filosóficas entre 'matar' y 'dejar morir', 'eutanasia activa' y 'pasiva', 'voluntaria', 'no voluntaria' e 'involuntaria', distinciones que tampoco emergen durante la discusión.

La apertura, por su parte, se produce cuando se distribuyen los roles, a saber, entre un sicólogo, un sacerdote, un diputado, un representante de los enfermos incurables, un médico y un padre de familia. Asimismo, está presente cuando se declaran los puntos de partida comunes: "la eutanasia permite evitar el sufrimiento de los pacientes y parientes y, por ello, hay que utilizar todos los medios que tiene la medicina" y "la eutanasia es una compasión falsa y con ella se evita que el individuo tenga el dolor purificatorio y meritorio que esta supone".

En la etapa de argumentación, los argumentos a favor de la legalización de la eutanasia en Chile solo se relacionan con el sufrimiento de los pacientes y sus familias y las consideraciones financieras, mientras que los argumentos en contra ponen el acento en la santidad de la vida humana, como un don de Dios, y se trata de defender, sin mucha convicción, el sentido religioso del sufrimiento como una experiencia purificadora. Los participantes usan términos arcaicos como 'meritorio' y 'purificatorio', sin explicación ni elaboración de estos conceptos. El tema del dolor emocional como una preparación para una muerte digna (Peck, 1999), indirecta o inconsciente, está ausente en los argumentos de quienes están contra la legalización de la eutanasia.

Un movimiento adecuado es el contra-argumento que distingue entre 'desconectar' y 'matar', en orden a mantener que la eutanasia no es un homicidio. Este planteamiento nos aproxima a la distinción entre eutanasia activa y pasiva, pero muestra que esta distinción, que podría haber sido muy útil para los argumentadores, no se encuentra en su repertorio. 
Lo anterior se aplica también al contra-argumento acerca de la prolongación (artificial) de la vida, que no sería una acción divina, sino humana. La distinción entre medios 'proporcionados' y 'desproporcionados' está latente aquí, aunque los estudiantes demuestran su desconocimiento de esta materia, lo que les impide utilizarla adecuadamente.

Los problemas pragmáticos solo se visualizan desde la perspectiva de los gastos que significa mantener artificialmente con vida a una persona que no tiene la posibilidad de sanarse y se sugiere que ese dinero podría utilizarse en programas de salud para aquellos que sí pueden acceder a dicho beneficio. Existe también una ignorancia acerca de las políticas del gobierno de Chile en relación con la eutanasia, desconociendo los proyectos de ley que se están gestando en el Congreso y cuál es la postura que prima en ese ámbito.

Por último, el cierre no aparece, lo que significa que el resultado de la discusión queda indeterminado.

\section{Comentarios finales}

El estudio acerca de cómo se desarrolla un debate simulado en torno a la legalización de la eutanasia en Chile con la participación de un grupo de estudiantes de tercer año medio, siguiendo el modelo analítico de la pragma-dialéctica, permite concluir, por una parte, que, en este caso, los estudiantes no cumplen con todas las etapas propuestas para una 'discusión crítica'. En otras palabras, no se logra resolver una diferencia de opinión mediante los intercambios verbales que deberían corresponder a los pasos metódicos de dicha discusión. Por otra parte, las argumentaciones allí esgrimidas no están siempre sustentadas en principios éticos y pragmáticos relacionados con la problemática en cuestión, ya sea por desconocimiento o por la poca implicación de los participantes en ella.

Esto no significa negar que una reflexión de esta naturaleza sea posible entre los jóvenes, pero el enfoque debe ser muy diferente: el entorno ideal para la educación ética es la 'comunidad de indagación', tal como es practicada por muchos profesores y estudiantes alrededor del mundo (Vicuña, 1999). Si los estudiantes se involucran más vitalmente, quizás a través de una película o un trabajo literario, puede ser posible crear un adecuado contexto para una discusión en dicha comunidad. Además, la misma comunidad permitiría practicar el 'código de conducta para los argumentadores razonables' que van Eemeren y Grootendorst (2004) recomiendan y, de ese modo, alcanzar con una comprensión mayor un resultado más coherente y conclusivo.

Más aún, lo anteriormente expuesto no es posible si no se cuenta con el apoyo del profesor frente a temas éticos complejos. Dicho apoyo deberá materializarse en la entrega no solo de herramientas conceptuales, sino también en la elección de un enfoque acerca de la argumentación 
que asegure una adquisición sólida de la competencia argumentativa en la enseñanza básica y media. De esta manera, podremos contribuir a un mejoramiento de la calidad de la participación democrática y del debate público en nuestra sociedad.

\section{REFERENCIAS BIBLIOGRÁFICAS}

Anscombre, J. \& Ducrot, O. (1976). L'argumentation dans la langue. Langages, 42, 5-27.

Feteris, E. (2007). Euthanasia and the teaching of argumentation in Chile. Comentario a ponencia presentada en The Ontario Society for the Study of Argumentation, Universidad de Windsor, Canadá.

Kuhse, H. (1995). La eutanasia. En P. Singer (Ed.), Compendio de Ética (pp. 405-416). Madrid: Alianza Editorial.

Lipman, M., Sharp, A. \& Oscanyan, F. (1980). Philosophy in the classroom. Philadelphia: Temple University Press.

MINEDUC (1998). Curriculum de la educación media. Objetivos fundamentales y contenidos mínimos obligatorios. Santiago de Chile: Ministerio de Educación de Chile.

Peck, M. (1999). La negación del alma. El problema de la eutanasia. Buenos Aires: Emecé.

Perelman, Ch. \& Olbrechts-Tyteca, L. (1958). La nouvelle rhétorique: Traité de l'argumentation. Bruxelles: L'Université de Bruxelles.

Seper, F. (Dir.) (1980). Declaración 'lura et bona' sobre la eutanasia. Ciudad del Vaticano [en línea]. Disponible en: http://www.vatican.va/roman_curia/congregations/cfaith/documents/rc_ con_cfaith_doc_19800505_euthanasia_sp.html

Toulmin, S. (1958). The uses of argument. Cambridge: Cambridge University Press.

Van Eemeren, F. \& Grootendorst, R. (1992). Argumentation, communication and fallacies. A pragmadialectical perspective. Hillsdale, NJ: Erlbaum.

Van Eemeren, F. \& Grootendorst, R. (2004). A systematic theory of argumentation. The pragma-dialectical perspective. Cambridge: Cambridge University Press.

Van Eemeren, F. \& Grootendorst, R. (2006). Argumentación, comunicación y falacias. Una perspectiva pragma-dialéctica. Santiago: Ediciones Universidad Católica de Chile.

Vicuña, A. M. (1994). Filosofía para niños: Algo más que desarrollar habilidades de razonamiento. Pensamiento Educativo, 15, pp.177-195.

Vicuña, A. M. (1999). Ethical education through philosophical discussion. Thinking, 14, 23-26.

Vicuña, A. M., López, C. \& Tugendhat, E. (1997). La fundamentación ética de los Derechos Humanos y algunas proyecciones para una educación en el respeto a todos los hombres. Informe final del proyecto FONDECYT 1940687, Santiago de Chile, Chile. 


\section{ANEXO \\ Transcripción de una clase acerca de la legalización de la eutanasia en Chile}

1. P: A ver | eh | ¿Quedó claro en este caso?| Por lo que decía la Javiera | estamos hablando de un texto de tipo litera:: rio | ¿sabe o no? por lo tanto | la opinión que ustedes tengan respecto de lo que ella plantea I jes correcta! | o sea | cada uno de ustedes | incluso yo misma puedo tener una opinión diferente | pero como es un texto literario y ella en un minuto lo dijo subjetivo::(f) || vale $\neg$ ¿de acuerdo? || alguna eh | pregunta al respecto | ¿algo que no quedó claro de esta disertación? | ¿o de esta presentación?

2. A1: ¿Podríamos ver la primera hoja?

3. P: ¿Cuál primera hoja?

4. A1: De la primera

5. P: A ver | hagamos lo siguiente | para que avancemos $\neg$ ¿tú puedes?...- | las hacemos correr || los que tienen y los que tengan =otras transparencias las hacen correr $=$

6. $\mathrm{A} 2$ : $=$ Yo quiero dar una opinión $=$

7. P: ¿Ah?

8. A2: Yo tenía una opinión que me pareció válida

9. P: ¿Una opinión?

10. A3: Una opinión de algo que salió

11. P: Marcela, pásale los apuntes a Sebastián

12. A3: (??)

13. P: Mantiene / el mismo tema pero ahora lo vamos a ver en la modalidad de I un debate | ellos van a simular un debate televisivo:: Tenemos al moderador || que él nos va a presentar a los participantes en este debate II

14. (Ruido)

15. P: Ya!

16. A1: Ya? || Hola | Muy buenas tardes a los panelistas | $\mathrm{y}:$ : a todos los televidentes que nos sintonizan hoy II eh | bienvenidos al barómetro || eh | hoy | como tema principal:: el tema que hablaremos es la eutanasia I eh | así como pequeña introducció::n | es el acto de acabar con la vida de una persona a petición o no a petición de ella con el fin de minimizar el dolor que esta tiene debido a la enfermedad terminal que posee II bueno y para hablar de este tema tenemos | estamos con los siguientes panelistas | a mi derecha se encuentra la sicóloga | Jocelyn Martínez | gracias por venir

17. (RISAS)

Mueve las manos en círculo

La profesora toma del brazo al moderador

El moderador se mueve 
18. A1: También está con nosotros el el el secretario general de la diócesis (ac) de Valparaíso don Bryan Zamorano II También nos acompaña hoy el diputado del PPD eh don Rodrigo Segovia | eh eh también por este otro lado tenemos al director general de una agrupación de personas | que ocuparon la eutanasia y están muy arrepentidos | Javier Vergara (RISAS) También nos acompaña hoy el director general del hospital Van Buren eh don Daniel Zavala || y por último también está un padre de familia (RISAS) un padre de familia don Camilo Sepúlveda || Bueno | y para:: discutir este tema II para discutir este tema les planteo la siguiente pregunta ise debe:: legalizar la eutanasia en Chile? Bueno | eso es de lo que hablaremos pero | primero debemos conocer las opiniones que tiene cada uno acerca del tema | bueno eh señorita Jocelyn (ac) | ¿qué opina usted acerca de la eutanasia?

19. A2: Mi opinión sobre la eutanasia es que es un mejoramiento de la calidad de vida a punto de evitar el sufrimiento ya sea para los pacientes o para los parientes eh I y por eso es importante utilizar todos los medios que tiene la medicina para evitar este sufrimiento

20. A1: Y Zamorano, usted ¿qué opina acerca de la eutanasia? Desde su punto de vista como iglesia 1

21. A3: Bueno eh | yo como sacerdote | pienso que la eutanasia I es una compasión falsa contra el contra el individuo que se está:: matando | y pienso que también eh es evitarle es evitarle el | dolor purificatorio y meritorio que tienen a la vez esta ehm

22. A1: $Y$ usted diputado I ¿qué piensa?

23. A4: Yo encuentro que | se está gastando mucho dinero innecesariamente | en personas que debiera ser utilizado:: más bien por ejemplo en el plan AUGE faltan todavía muchas enfermedades por ser integradas y se está gastando mucho dinero en personas que | ya no tienen vuelta atrás | que están viviendo agónicamente y debería ser gastado mejor en personas que sí tienen posibilidades de seguir viviendo I y que hay gente que muere por no tener los recursos suficientes | para pagar su tratamiento

24. A1: ¿Usted que tiene un gasto innecesario? II y ¿usted Javier qué opina?

25. A5: Yo opino que | no se debería legalizar la eutanasia | por que luego de practicarla lo más probable es que:: la persona se arrepienta I y esto no hay forma de revertirlo y esto después puede traer un trauma sicológico para la

El moderador mira a Jocelyn

A2 se rasca la nariz

A3 mueve la mano

(Bulla de ambiente)

A4 sonríe

A5 mueve sus dedos

A5 asiente con la cabeza 
26. A1: Así | algo así | como remordimiento

27. A1: Y:: Daniel Zavala | desde el punto de vista de los hospitales ¿qué I piensa usted?

28. A6: Yo creo que | la eutanasia sería una gran ayuda | para:: los hospitales y sus enfermos | debido a que | los enfermos terminales ocupan | mucho mucha muchas cami |camillas que podrían ocuparse para enfermos que tienen salvación para que | se salven $\backslash$

29. A1: ¿Y por último | don Camilo Sepúlveda | qué opina usted?

30. A7: Yo creo que la eutanasia es un I procedimiento que se ve desde el punto de vista profesional | de los médicos | $\mathrm{y}::$ no se ve desde el lado afectivo y sentimental

31. A1: Conocidas todas las opiniones | ahora iremos con el debate I que se inicia a través de (??) ¿se debe legalizar la eutanasia en Chile? I esta pregunta se la hago a usted señorita Jocelyn I ¿qué opina?

32. A2: Desde mi punto de vista la eutanasia es una muerte provocada | por:: propia voluntad y sin sufrimiento físico | es una muerte a un enfermo incurable y esto se denom | denomina como muerte por compasión.

33. A7: No estoy de acuerdo con usted porque | detrás del:: enfermo | se encuentra una familia | y: | la familia sufriría si | por ejemplo | yo matara a mi hijo que está terminal I

34. A5: Sí | yo estoy de acuerdo con él | porque conozco la situación (ac) de otra familia que le hicieron eutanasia y ellos saben lo que es | se sienten culpable ahora ||

35. A6: El:: el hospital Van Buren | es un hospital público | en el que existen una variedad de enfermos no terminales । que tienen salvación/ | debido a la falta de dinero | eh no pueden ser operados |0:: o comprar sus remedios | eh

36. A7: Pero se supone que | la familia está pagando para | para mantener a su hijo postrado en una cama

37. A4: Yo voy a dar mi opinión I yo encuentro que cuando se habla de que se está matando una persona I cuando se desconecta por ejemplo de una máquina | yo encuentro que no se está matando al contrario se está alargando la vida de las personas cuando no está conectada la máquina | por que si |por ejemplo | si nos remontáramos cien años atrás | una persona que | una persona que está un enferma terminal | no tendría la tecnología de hoy en día y moriría no más poh | fallecería de forma natural | y hoy en día se está alargando las vida de las personas y muchas veces están:: agónicos | y están sufriendo y la familia sufre también $\backslash \| \mid$
Mueve una de las manos que está apoyada en una mesa

A7 está sentado con los brazos cruzados

A1 mira directamente a Jocelyn

A5 apunta a A7 y se dirige a A2

Mueve los brazos y los vuelve a cruzar

A4 se dirige a A7 
38. A3: Eh | yo creo que la eutanasia | eh pa mi perspectiva | yo creo que la eutanasia incide a la muerte | y como Dios dice | desde muy:: tiempo antiguo | dice:: nos da diez mandamientos | los cuales dicen | uno de ellos | dice no matar I y eso es lo que está haciendo la eutanasia | estai matando | es un asesinato

39. A6: Mira | la medicina lo único que hace | es fue creada por el hombre | es alargar la vida | por nuestra voluntad | entonces Dios nos dio una vida hasta que tu cuerpo no da abasto | entonces hasta ahí debería ser lo que usted dice I pero nosotros la alargamos porque queremos porque no queremos morir $\backslash$ es un consentimiento \}

40. A2: Lo cual no se debe | no se debe: intentar prolongar la vida | cuando esté ya no se puede vivir | haciendo que ya el paciente | ya no sea un ser humano | o sea no sea un ser humano y pase a ser un caso | por ejemplo de una clínica | como lo es hoy en día en la medicina

41. A3: Eh tú ¿para qué? | eh por ejemplo una persona | ipara qué practica la eutanasia? | eh la:: eutanasia se practica solamente pa sacarse un cacho II porque también lo vemos por el lado del aborto | el aborto es (??) ¿porqué? | bueno pa sacarse un cacho | la eutanasia pasa lo mismo | para sacarse un cacho || al final | esto | por el lado de la iglesia | Dios te dio la vida | y la vida es para vivirla | vivimos para vivir | y eso eso es lo que no se está lo que no se está respetando | porque estamos matando a alguien que verdaderamente tienen que dejar vivir | no si | la vida termina cuando Dios quiere II

42. A1: ¿Y usted Camilo que ha vivido esta experiencia de tener un hijo de enfermo terminal? ¿qué opina de lo que dice Zamorano?

43. A7: No | totalmente de acuerdo en lo que ...- yo creo que | le digo a ellos que quieren a pedir eutanasia | tiene que ponerse en los zapatos de las personas que sufren ese mal y que siempre ven la parte profesional y no los sentimientos

44. A1: ¿0 sea lo que quiere decir que ellos ven sólo la parte del dinero y no la parte del amor que usted siente por el enfermo? ¿eso es lo que quiere?

45. A7: No tanto el dinero | sino como la parte (??)

46. A6: pero ¿usted sabe que:: | usted podría salvar muchas vidas | ocupando la eutanasia? | usted podría donar órganos a gente que lo necesita | y esos remedios que se ocupan en

A3 se dirige a $A 2$

Se rasca la cabeza y mueve las manos

\section{A7 permanece de brazos cruzados}

A7 con los brazos cruzados mueve ellos | se podrían ocupar en otra gente ||

una mano

47. A7: Pero la familia sufre 
48. A6: La familia sufre pero | pero deberían estar contentos porque él || va a vivir | va a vivir en otras partes | o sea si | porque están sus órganos en otra vida ।

49. A2: También la eutanasia explica que un médico | mate por:: medios dolorosos a un paciente | que sufre de un dolor físico y sicológico | y luego | o sea | esta muerte puede ser digna | porque la familia y el paciente están sufriendo | | y para qué | para qué seguir con el sufrimiento si se puede evitar por medio de la eutanasia

50. A3: Eh |el dolor que dice | el dolor | según el lado de la iglesia | el dolor está:: visto no como | oh está sufriendo | el dolor está visto como:: || un eh mm | participación con la pasión de Cristo(ac) | el dolor purifica el alma || el dolor también | eh | es como algo de meditación | ¿ya?| eh según el lado de la iglesia el dolor es una participación con Cristo | con la pasión que él tuvo

51. A6: Pero ¿para qué más dolor I que tuvo durante toda su vida poh? I ¿para qué darle más dolor cuando está muriendo? | si ahí el cuerpo ya no da más | sufrió todo esto durante la vida I ¿pa que darle más dolor?

52. A5: El dolor purifica el alma

53. A6: ¿Pa qué purificarla más? Si ya está listo | no hay nada más que hacer | $\mid$

54. A2: Se está enfermando él ly el que está como en estado vegetal | y está enfermando a la | a la misma vez a la familia | porque la familia está viendo cómo sufre ese paciente I y ese es un dolor sicológico que puede ser igual o más importante que el dolor físico

55. A6: La iglesia | la iglesia también |apoya | es decir | tiene que evitar la pobreza | tiene que ayudar a los pobres | porque:: por eso la:: eutanasia lo podría ayudar I porque mira hay gente I hay familias que ocupan mucho dinero para:: enfermos terminales | y quedan en la ruina | a lo mejor eso aumenta la pobreza
A6 se dirige a $A 7$ y mueve una hoja de papel que tiene en la mano

A 2 mueve las manos, lee un papel y se toca el pelo de vez en cuando

A6 se dirige directamente a $A 3$ 